\title{
El feminismo y los estudios de género
}

\author{
Adriana Renné Tobos Vergara ${ }^{1}$, Ángela Paola Ochoa ${ }^{2}$, Lizeth Cristina Martínez Baquero ${ }^{3}$, \\ Liliana Paola Muñoz Gómez ${ }^{4}$ \& Mildred Alexandra Vianchá Pinzón ${ }^{5}$
}

\begin{abstract}
This article reveals a reflection on the emergence of gender studies linked to the ideas of feminism. It starts with a brief definition about the concept, as evidenced in the historical overview of countries such as England, France and United States, as well as the main approaches and achievements established over time; subsequently settles the differentiation of the category gender as a starting point in the study of women, masculinity and the subsequent development of the Queer theories. Finally highlights the importance of gender in the approach to sexuality studies.
\end{abstract}

Editado por | William Alejandro

Jiménez-Jiménez,

Universidad de Boyacá, Colombia.

Revisado por |Ana María Ávila-

Matamoros,

Fundación Universitaria Konrad

Lorenz, Colombia.

Keywords | feminism, masculinity, queer theory, sexuality, gender.

\section{Introducción}

¿De qué manera el feminismo se ha configurado como un movimiento social?, ¿̇el feminismo busca la superioridad de la mujer sobre el hombre?, ¿los estudios de género giran en torno a

\author{
${ }^{1}$ Adriana Renné Tobos Vergara \\ $[\bowtie]$ \\ ${ }^{2}$ Ángela Paola Ochoa
}

${ }^{3}$ Lizeth Cristina Martínez Baquero

${ }^{4}$ Liliana Paola Muñoz Gómez

${ }^{5}$ Mildred Alexandra Vianchá Pinzón
Psicóloga - Universidad Pedagógica y Tecnológica de Colombia. Magíster en Psicología. Universidad Nacional de Colombia.

Estudiante de Psicología Universidad de Boyacá. Integrante del semillero PAIDEIA

Psicóloga Universidad Pedagógica y Tecnológica de Colombia. Máster en Psicología, Clínica A.E.P.C. Directora de Investigaciones, Facultad de Ciencias Humanas y Educativas. Email: Icmartinez@uniboyaca.edu.co

Psicóloga - Universidad Cooperativa de Colombia. Magíster en Docencia, Universidad de La Salle. Docente Universidad de Boyacá.

Psicóloga - Universidad Católica de Colombia. Magíster en Psicología. Directora del Programa de Psicología - Universidad de Boyacá. Email: maviancha@uniboyaca.edu.co 
la masculinidad?. Éstas son algunas de las preguntas que surgen en torno a planteamientos feministas fundamentales y las cuales dan cuenta de la re significación que la mujer ha tenido frente a un cambio social y cultural a lo largo de muchos siglos.

El aporte del feminismo se vislumbra más allá de una simple participación en aspectos políticos o sociales; ya que han logrado permear disciplinas que se han interesado en desarrollar estudios desde perspectivas de género, llegando a establecer nuevas miradas acerca de la feminidad y la masculinidad. Así, poco a poco se han adentrado en la objetivación de géneros disidentes que empiezan a encontrarse con mayor frecuencia en los estudios actuales.

Pensar en feminismo suele evocar en las personas, aspectos relacionados con el lesbianismo, la lucha por los derechos, la obtención del voto popular en las mujeres, la búsqueda de la igualdad, y mujeres masculinizadas que se oponen a los hombres con actitudes extremistas. Estos significados atribuidos por la sociedad reflejan el impacto, favorable o tergiversado que ha tenido el feminismo, por lo que antes de avanzar en la reflexión, se hace necesario precisar algunas definiciones de este concepto.

\section{El feminismo}

El término empieza a usarse en Francia (1880) y más tarde surge en Estados Unidos (1919), como respuesta a la búsqueda de otras miradas sobre el protagonismo de la mujer, objetivando su realidad a través del reconocimiento de aportes que ha hecho esta a lo largo de la historia al igual que los logros alcanzados en las sociedades pese a su invisibilización.

El planteamiento principal del paradigma feminista basa su formulación en la crítica y el rechazo a la postura androcentrista, visión del mundo que sitúa al hombre como centro de todas las cosas, denunciando que bajo esta mirada se han plasmado los descubrimientos científicos y los planteamientos más importantes recordados por la humanidad, los cuales de una u otra forma han dejado de lado el reconocimiento femenino (Butier, 1990). El surgimiento del feminismo se relaciona comúnmente con la aparición del movimiento de liberación femenina de los años 70 en Estados Unidos, establecido a partir de un amplio desarrollo histórico y social.

Autores como Badinter (1993) resaltan el surgimiento de las primeras expresiones feministas (Francia e Inglaterra) como consecuencia de deseos de emancipación y cambios de los valores 
tradicionales surgidos para esa época. En Francia, las feministas buscaron ascenso en la posición social, derecho a la dignidad, a la educación y cuestionaron la institución del matrimonio en búsqueda de la libertad pero sin renunciar al amor. Por el contrario las feministas Inglesas parecieron exigir libertad, igualdad, goce sexual y a no ser abandonadas en el embarazo.

Estos planteamientos conllevaron a un cambio en la concepción de la masculinidad, principalmente al punto de imposición de valores femeninos frente al desdibujamiento de los valores masculinos a través de la desaparición del "dualismo oposicionista que caracteriza al patriarcado, para cederle paso a una especie de unisexismo" (Badinter, 1993, p. 31).

Estudiosos de la historia advierten tres momentos importantes en el desarrollo de los planteamientos paradigmáticos feministas, a los que denominan olas del feminismo. La primera ola se encuentra estrechamente relacionada con la Revolución Francesa (1789) la cual vincula el surgimiento del feminismo con la búsqueda de derechos jurídicos y la designación del espacio privado en el hogar, generando una discusión en el reconocimiento del papel desempeñado por las mujeres en la sociedad. Sin embargo, esto no obtuvo grandes logros. A partir de allí las mujeres francesas tomaron conciencia de su exclusión y opresión por parte del estado, de esta forma se proclamó una fuerza de búsqueda de derechos mediante la movilización encaminada hacia la consecución de las luchas (Maldonado, s.f; Kogan, 2008)

La segunda ola aparece con la exigencia del derecho al voto femenino logrado a principios del siglo XX inicialmente en España e Inglaterra y progresivamente en la mayoría de países desarrollados; tras la partida de los hombres a las guerras. Fue entonces cuando las mujeres se encargaron de sostener la economía familiar y plantear otras luchas como el acceso a la educación superior, compartir la patria potestad de los hijos con el esposo y la búsqueda de igualdad de salarios; esto generó un reconocimiento a nivel social.

La tercera ola del feminismo surgió entre los años sesentas y ochentas. En esta época surgió el inconformismo de las mujeres al ser definidas como esposas y madres dedicadas al hogar. Es allí donde se constituye el Movimiento de Liberación, el cual manifestó un papel protagónico de la mujer en el ámbito laboral. Ésto trajo grandes cambios respecto a la concepción que se mantenía sobre la feminidad y la participación de la mujer en actividades políticas, sociales y económicas (Maldonado, s.f).

$\boldsymbol{\epsilon} \| 60$ 
Como se evidencia, el feminismo ha tenido un desarrollo histórico, por lo que se debe evitar debates feministas malinterpretados que deforman el significado real del movimiento (Castellanos y Accorsi, 2001).

De esta manera, el alcance de las discusiones en torno a teorías feministas y la comprensión de las mismas han tenido un extenso auge y reconocimiento para la comunidad científica ya que el desarrollo de los estudios de género como la masculinidad o las teorías Queer se han establecido ampliamente, creando así aportes significativos y un interés mayor en la comunidad científica al momento de realizar análisis de las problemáticas que afectan no sólo a hombres y mujeres, sino a las sociedades en general.

Es así como estos aportes investigativos han volcado la mirada hacia el estudio y exploración de temas particularmente interesantes como el ejercicio de la sexualidad, la participación de las mujeres en la vida política, el digno acceso de las mujeres al ámbito laboral, la maternidad compartida, la erradicación del machismo, la masculinidad, entre otros.

\section{La categoría género en los estudios}

Sorprendentemente la mirada feminista puso en relieve la necesaria diferenciación entre la categoría género y la categoría sexo. Por lo tanto, en este apartado se hace especial énfasis en la categoría género.

Los primeros estudios que se realizaron sobre el género tienen su origen hacia los años sesenta, época en la que surgieron movimientos sociales que cambiaron la manera de comprender el significado y rol de ser hombres y mujeres, los cuales fueron propiciados por los movimientos feministas (Lamas, 1999).

Estos estudios corresponden a marcos teóricos e investigativos que reconocen la existencia de distintos géneros (masculinos, femeninos, andróginos, homosexuales y lésbicos) en los que se visibilizan necesidades, problemáticas y discursos en búsqueda del reconocimiento, derechos y deberes que reflejen equidad con el fin de establecer diferencias significativas entre categorías género y sexo se hace indispensable. 
De esta manera Lamas (1999) advierte que bajo la categoría género se encuentra inmerso el estudio de las construcciones sociales que varían en la concepción del ser masculino, femenino, andrógino u homosexual; mientras que en la categoría sexo se enfatizan ciertas características biológicas que agrupan las categorías hombre y mujer.

En cuanto a la categoría de género cabe resaltar el inicio de su significado, al ser incluido en los discursos de las ciencias sociales solo hasta 1995 por Money quien empleó el término para referirse a las conductas atribuidas a hombres y mujeres, dando especial énfasis a las diferencias socioculturales y menos a aspectos biológicos entre unos y otros, diferencias que marcan a su vez pautas relacionales particulares que posibilitan la construcción de identidad de hombres y mujeres (Lamas, 1999).

Para analizar la categoría género es necesario revisar a profundidad no sólo las construcciones sociales o las relaciones de poder; sino la asimetría entre géneros, la implicación de otras instituciones a nivel social, los símbolos y sistemas económicos y políticos; con el fin de proponer soluciones para la búsqueda de equidad (Gamba, 2008).

El abordaje de los estudios de género es trascendental para los diversos espacios de socialización humana como lo son la educación, la construcción de identidad, las oportunidades laborales, entre otras; ya que se requiere una reflexión más profunda de aquello que significa e implica ser hombre o ser mujer a través de la construcción de identidades sexuales.

Precisamente bajo la mirada de las posturas de igualdad y diferencia se ha analizado el concepto de género que surge de discursos no científicos; aunque para muchos analistas su desvalorización y poco interés. Dichos discursos fueron fomentados por los movimientos desde las vertientes de la igualdad y de la diferencia las cuales conciben la existencia de diferencias substanciales de orden sociocultural entre los hombres y las mujeres (Comas, 1995).

Sin embargo, planteamientos más recientes sugieren que así como en un principio el feminismo denunció la existencia de una hegemonía patriarcal, también se ha visibilizado la hegemonía de los géneros masculino y femenino. En la actualidad se ha centrado la discusión en el reconocimiento de otros géneros que suelen hacer presencia con mayor fuerza en los estudios de género incluidos en las teorías Queer.

\section{E $\| 62$}


Es importante entonces que dentro de la categoría género se explore y estudie más sobre temas directamente relacionados como la masculinidad, feminidad y homosexualismo (Mouffe, 1993). Se podría decir entonces que género es una construcción social que varía según el espacio y la temporalidad, que denota diferencias entre los integrantes del mismo género y que necesariamente parte de las diferencias biológicas y socioculturales entre hombres y mujeres.

Así, Lamas (1999) plantea el término género como un elemento básico de la construcción de la cultura, el cual denota los roles sexuales otorgados a partir de la división biológica y orgánica. Además de la diferencia en la participación de los hombres y las mujeres en instituciones sociales, económicas, políticas y religiosas, actitudes, valores y expectativas que una sociedad determinada conceptualiza como femenino y masculino.

De igual manera, Lamas (1986) formula que las sociedades piensan sus propias divisiones internas donde se categorizan las distinciones entre hombre - mujer o macho - hembra adjudicando a esta última mayor cercanía con la naturaleza al vincularle la función reproductora como un hecho cultural.

Es así como el objetivo científico de los planteamientos feministas se encaminó a la comprensión de la realidad social de las mujeres a través de la búsqueda de reconocimiento y distinción política tanto en hombres como en mujeres, es decir más allá de considerar lo femenino como una condición natural del sexo buscó señalar la construcción del ser mujer como un complejo proceso individual y social.

Esta legitimación y reconocimiento facilitó que a finales de los años 80 's las estudiosas feministas sustituyeran la palabra mujeres por género. Para Scott (1996) la utilización de la categoría género aparece no sólo como la forma de hablar de los sistemas de relaciones sexuales o sociales; sino también como forma de situarse en un debate teórico.

Mediante el proceso de constitución de orden simbólico en una sociedad se fabrican las ideas de lo que deben ser los hombres y las mujeres; ya que los planteamientos feministas sobre las consecuencias de la diferenciación sexual, han captado las redes de significados alrededor de las categorías sexo y género para comprender cuáles son las estructuras de poder que dan forma al modelo dominante de sexualidad: la heterosexualidad (Lamas, 1999). 


\section{El surgimiento de los estudios de masculinidad}

Cuando los estudios feministas hicieron evidente la situación social de las mujeres, muchas miradas centraron la atención en el estudio de los hombres. Esto dio como resultado la discusión alrededor de los sexos al establecer un orden social, distribuir los roles de sus individuos y diferenciar entre lo masculino, lo femenino, el hombre y la mujer, tanto a nivel simbólico como a nivel relacional (Parranini, 2002).

Es por eso que al incluirse la categoría "género" hacia los años 80 's por parte del feminismo anglosajón se privilegió una nueva perspectiva cultural en la diferenciación entre masculino y femenino como un "status sexual” (Arango, León y Viveros 1998).

La masculinidad se define entonces, como un status a lograr y ciertas cualidades a desarrollar por medio de pruebas y del modelamiento de la sensibilidad de los niños educados por la madre para volverse hombres, (Olavarría, 2003). Elías y Duning (1986) sugieren que la cultura masculina se desarrolla dentro de los grupos de pares, en la cual es necesario exagerar los rasgos viriles como forma de afirmación sexual, implicando también la actitud de denigrar a la mujer. Sin embargo, en muchos estudios de esta clase se hacen generalizaciones de hallazgos encontrados en grupos de "chicos malos" implicados en conductas delictivas.

Desde una perspectiva conservadora, la masculinidad fue considerada un atributo natural y/o divino esencial a los hombres y fundamental para la sobrevivencia de la especie humana. Otros aportes como el de Kaufman (1995) sugieren al poder como el elemento central de la subjetividad masculina justificado a su vez en la dominación sobre las mujeres y además el poder colectivo interiorizado, individualizado, encarnado y reproducido por el masculino.

Los estudios que tuvieron como protagonistas a los hombres iniciaron su amplia trayectoria en Inglaterra, Estados Unidos y América Latina, sugiriendo una crisis de la masculinidad caracterizada por la existencia de múltiples masculinidades. Esta crisis en palabras de Badinter (1993) plantea una necesidad de cambio en respuesta a las exigencias que imperan tras los cuestionamientos surgidos a partir de los alcances de los movimientos sociales de mujeres y el feminismo.

Posturas antifeministas que representan nuevos puntos de vista como el análisis de Badinter (1993) refiere que la crisis de la masculinidad es causa de lo que implica ser hombre y como eso 
se configura de una manera más compleja que el ser mujer. En el mundo moderno se ha dado explicación a realidades contextuales como la violencia intrafamiliar, el divorcio, el aborto, las relaciones conyugales y las diferencias entre hombres y mujeres para cada caso. Sin embargo, estos son tradicionalmente considerados de índole femenino donde actualmente protagonizan los estudios de masculinidad. Estos planteamientos constituyeron un desafío teórico y empírico al investigar la masculinidad como una construcción cultural y a los hombres como sujetos particulares (Parranini, 2002).

A finales de la década de los ochentas incursionaron los estudios de masculinidad abordados desde reflexiones históricas, posturas antropológicas y estudios sobre la relación existente entre masculinidad, teorías sociales y corrientes mitopoéticas (Kimmel, 1993). Estudios realizados, especialmente en México, han encontrado que los hombres afirman su masculinidad mediante el ejercicio de la fuerza, el valor, la protección y el poder además de la constante necesidad de probar el ser hombre especialmente en el ámbito sexual (Tuñón y Eroza, 2001).

Otras investigaciones sobre la construcción social de la masculinidad realizadas en Chile especialmente, plantean la existencia de un modelo hegemónico de masculinidades, es decir que éste es un elemento estructurador de las identidades individuales y colectivas del continente americano al centrarse principalmente en pautas de identidad afectivas, y comportamentales, además de la experiencia subjetiva de ser hombre (Fuller, 2001; Olavarría, 1998; Viveros, 1997; Ramírez, 1997)

La Psicología como ciencia hace un aporte significativo al estudiar la masculinidad como construcción social, más allá de las concepciones netamente biológicas frente al género ya que los estudios de masculinidad responden a la necesidad política y teórica de reconocer y especificar las dinámicas del sistema y señalar el modo en el que se configura el fenómeno (Parranini, 2002). En Colombia, por ejemplo los estudios de género se han enfocado especialmente en las situaciones alrededor del conflicto armado (Ayín, 2003). El estudio de las masculinidades en las zonas rurales ha sido poco estudiado y solo recientemente, a raíz de la reforma agraria, se ha realizado un abordaje, esto se evidencia en estudios como los de Neira y Ruiz (2001).

Los retos que enfrentan los estudios de masculinidad están centrados especialmente en la profundización del estudio de un modelo más comprensivo acerca de las nuevas realidades sociales 
como: el travestismo, la homosexualidad, la heterosexualidad, y otros géneros no hegemónicos, además de los que Parranini (2000) podría denominar como masculinidad guerrera, sostenida y avalada en instituciones como las Fuerzas Armadas o grupos sociales emergentes como las pandillas juveniles o las barras bravas.

\section{Más allá del ser hombre y ser mujer. El surgimiento de las Teorías Queer}

Tras plantear los estudios del hombre como legado del feminismo en respuesta o contraposición a éste, recientemente se han elevado voces que denuncian los estudios de género centrados en el interés hegemónico masculino y femenino.

Conforme a lo anterior, las teorías Queer, como elaboraciones teóricas disidentes, pretenden visibilizar la necesidad de abandonar la idea de sexualidades "normales" para adentrarse en el estudio de las sexualidades periféricas desde una perspectiva que dé cuenta de géneros socialmente aceptados. El movimiento Queer es un movimiento disidente de géneros basado en las normas que impone la sociedad heterosexual dominante (Hernández y Quintero, 2009)

En sus inicios, el término Queer servía para nombrar aquello que por su condición de inútil, mal hecho, falso o excéntrico; ponía en cuestión el buen funcionamiento del juego social y también a todo aquel que no pudiera ser inmediatamente reconocido como hombre o mujer. En la actualidad, Queer ha pasado a ser un signo de resistencia a la normalización a ser un indicador revolucionario (Hernández y Quintero, 2009). Mérida (2002) afirma que Queer hace referencia a la naturaleza subversiva y transgresora de una mujer desprendida de la femineidad subordinada, una mujer masculina, un hombre afeminado o con una sensibilidad contraria a la tipología masculina.

En relación con los estudios de género, el interés de los teóricos Queer plantea que la sexualidad no debe ser tomada simplemente como ser hombre, mujer, gay o lesbiana, sino como construcciones de la sociedad que al clasificar los géneros excluye a las personas que no se rigen a la norma establecida (Mareñu, 1996).

Hoy en día los estudios Queer marcan la pauta en el cambio de abordajes de los estudios de género, los cuales enriquecen el panorama que inicialmente planteó el feminismo.

\section{$\boldsymbol{\epsilon} \| 66$}




\section{Un vistazo final al legado de los estudios de género y sexualidad}

El abordaje de la sexualidad desde los estudios de género define el aspecto complejo de la construcción del mismo a través del desarrollo de procesos en los cuales los sujetos alcanzan una normatividad de la heterosexualidad y la re significación de la identidad sexual. Se toma un nuevo rumbo en los abordajes mostrando las diferencias entre los deseos que pueden tenerse, expresarse y satisfacerse dentro de un ordenamiento cultural (Fuller, 1995).

Por otra parte, Foucault (1994) define el término sexualidad desde la construcción del propio individuo, como aquel que intenta adentrarse en una sociedad moderna, cuyas fuerzas y estrategias escapan casi por completo del control individual y que involucra un juego de poder extremadamente particular, en donde el individuo puede optar por el enfrentamiento o acomodarse a las estrategias de normalización. Estos fenómenos se presentan en la sociedad junto con el despliegue y descubrimiento del género dentro de la identidad sexual, como parte fundamental en el desarrollo social del sujeto, por lo que se puede definir la sexualidad adoptando el postulado de Foucault.

En todo caso, género y sexualidad se encuentran enmarcados en el análisis de la diferencia significativa entre hombres y mujeres a través de la construcción de la identidad personal, a partir de un proceso cultural.

Este análisis permite tener claro que la sexualidad no define por sí misma la naturaleza, sino que involucra aspectos culturales, de dependencia, de cambios complejos y extraños reflejados en el concepto de género como consecuencia de la construcción individual, independientemente de femenino o masculino, sino mas bien el complemento de este dúo, en una sociedad moderna que cuestiona toda proporción, enfrentándose a un periodo que impide encontrar una salida y que obliga a la reflexión.

Los estudios de sexualidad desde una perspectiva de género permiten la comprensión de realidades particulares que permiten caracterizar y ejemplificar las relaciones de los sujetos sociales en contextos más amplios para establecer mayor claridad comprensiva frente a las formas de relacionarse consigo mismos y con otros, planteando respuestas a postulados que faciliten trazar rutas más acertadas y eficaces en la comprensión de problemáticas sociales que están presentes en la realidad y particularidad de los pueblos. 


\section{Notas de los autores y agradecimientos}

Este artículo hace parte de la investigación titulada: Caracterización de las Prácticas y conductas sexuales del uso del condón en jóvenes escolarizados de Boyacá.

\section{Referencias}

Arango, L., León, M., y Viveros, M (1998). Género e Identidad. Ensayos sobre lo femenino y lo masculino. Tercer Mundo: Santa Fe de Bogotá.

Ayín, N. (2003).Género en la Región Andina, aproximaciones y desafíos en torno a la Investigación. Cuaderno de estudios de Género. México. Instituto de estudios para la transición democrática. Recuperado de http://webkreator.com.mx/IETD/marta_lamas_curriculum.html.

Badinter, E. (1993). XY, la identidad masculina. Santa Fe de Bogotá: Editorial Norma. Traducción de Ana Roda.

Butier J. (1990). "Variations on sex and gender: beauvoir, witting, and foucault", en: Seyla Benhabib y Drucilla Cornell (eds.). Teoría feminista y teorípxrítica: Valencia.

Catellanos, G. \& Accorsi, S. (Comp. 2001). Sujetos femenimos y masculinos. La manzana de la discordia. Centros de Estudios de Género, Mujer y Sociedad. Universidad del Valle: Colombia.

Comas, D. (1995). Trabajo, género, cultura. La construcción de desigualdades entre hombres y mujeres. ICARIA: Barcelona.

Foucault, M. (1994). Historia de la Sexualidad (Tomo 1). México: Editorial Siglo XXI.

Fuller, N. (1997). Identidades masculinas, varones de clase media en el Perú. Fondo Editorial de la Pontificia Universidad Católica del Perú. Lima, Perú.

Fuller, N. (2001). Masculinidades, cambios y permanencias. Fondo Editorial de la Universidad Católica del Perú. Lima, Perú.

Gomáriz, E. (1992). Los estudios de género y sus fuentes epistemológicas: periodización y perspectivas. ISIS Internacional, Fin de Siglo, Género y cambio civilizatorio. Santiago de Chile. Ediciones de las Mujeres. Recuperado de http://entimema.pbworks.com/f/sobre+la+categor\%C3\%ADa+de+g\%C3\%A9neroMarta+Lamas.pdf. 
Keijzer de B. (1997). El Varón como factor de riesgo: masculinidad, salud mental, salud reproductiva, en Esperanza Tuñón (coord): Género y Salud en el sureste de México. El Colegio de la Frontera Sur.

Kogan, L. (2008). Estudios sobre sexo/género y cuerpo en el Perú. Espacio abierto, 17 (2), 285-299. Recuperado de http://www.redalyc.org/articulo.oa?id=12217205.

Lamas, M. (1986). La antropología feminista y la categoría "género". Nueva antropología, 8 (30), 173-198. Recuperado de http://www.redalyc.org/articulo.oa?id=15903009

Lamas, M. (1999). Usos, dificultades y posibilidades de la categoría género. Papeles de población, 5 (21), 147178. Recuperado de http://entimema.pbworks.com/f/sobre+la+categor\%C3\%ADa+de+g\%C3\%A9ne ro-Marta+Lamas.pdf

Lamas, M. (2000). Diferencias de sexo, género y diferencia sexual. Cuicuilco, 7 (18), 1-24. Recuperado de http:// www.redalyc.org/articulo.oa?id=35101807

Maldonado, C. (s.f). Las olas del feminismo... ¿Hasta dónde Ilegan?. http://www.biblicaemanuel.com/olas_feminismo2.htm.

Mareñu, A. et. Al. (1996). Géneros Prófugos: Feminismo y Educación. México.

Mouffe, Ch. (1993). Feminismo Ciudadanía y política democrática radical. Debate feminista: México.

Olavarría J., \& Parrini, R. (1998). Los padres adolescentes. FLACSO, Unicef, Santiago, Chile.

Olavarría, J. (2003). Varones Adolescentes, género, identidades y sexualidad en América Latina. FLACSO-Chile; INUAP; Red de Masculinidad.

Scott, J. (1996). El género, una categoría útil para el análisis histórico. En: James Amelang y Mary Nash (eds.), Historia y género: las mujeres en la Europa moderna y Contemporánea, Valencia, Ediciones Alfons el Magnánim.

Tuñón, E. \& Eroza, E. (2001). Género y sexualidad en adolescentes. La búsqueda de un conocimiento huidizo. Estudios sociológicos, 19(1), 209-226. Recuperado de http://www.redalyc.org/pdf/598/59855109.pdf

Viveros, M. (1997). Los estudios sobre lo masculino en Latinoamérica. Una producción teórica emergente. Nómadas, 6. Recuperado de http://www.redalyc.org/articulo.oa?id=105118999005. 


\section{El feminismo y los estudios de género}

Resumen | El presente artículo da cuenta del surgimiento de los estudios de género vinculados a los planteamientos del feminismo. Se inicia con una breve definición en torno a dicho concepto, tal y como se evidencia en la descripción histórica de países como Inglaterra, Francia y Estados Unidos, al igual que los principales planteamientos y logros establecidos a lo largo del tiempo; posteriormente se establece la diferenciación de la categoría género como punto de partida en el estudio de mujeres, la masculinidad y el subsecuente desarrollo de las teorías Queer. Finalmente se resalta la importancia de los estudios de género en el abordaje de la sexualidad.

Palabras clave | feminismo, masculinidad, teoría Queer, sexualidad, género.

\section{Citación}

Tobos-Vergara, A., Ochoa, A., Martínez-Baquero, L., Muñoz-Gómez, L., \& Vianchá Pinzón, M. (2014). El feminismo y los estudios de género. Enfoques, 1(1). pp. 58-70. 